\title{
Espacios de significación y representación de las teorías cognitivas, de la mediación y de la multirreferencialidad en el proceso de alfabetiza- ción hacía el aceso al conocimiento
}

\author{
Aida Varela* \\ Marilene Abreu Barbosa**
}

\section{Resumen}

Este artículo analiza las exigencias del siglo XXI en cuanto al acceso del hombre al conocimiento, destacando la dificultad de aprehender la realidad en un contexto de transformaciones científicas, tecnológicas, culturales, políticas sociales y económicas. Se revisan los conceptos de analfabetismo, alfabetización y la privación cultural y además la función de la escuela y de la biblioteca en el proceso de aprendizaje subsidiando el desarrollo del poder informativo. Reflexiona sobre las perspectivas de la organización y difusión del conocimiento, la mediación, la multirreferencialidad y la necesidad de políticas públicas para promover la mejora de la alfabetización informacional y comunicacional.

Palabras clave: acceso al conocimiento, la alfabetización, exigencias del siglo XXI, la mediación, la cognición.

Título: Espaços de significação e representação das teorias cognitivas, da mediação e da multi-referencialidade no processo de alfabetização frente o acesso ao conhecimento

\section{Resumo}

Este artigo analisa as exigências do século XXI, quanto ao acesso do homem ao conhecimento, destacando a dificuldade de apreender a realidade no contexto do progresso científico, tecnológico, cultural, social e das políticas econômicas. Analisam-se os conceitos de alfabetização, letramento e privação cultural e o papel da escola e biblioteca no processo de aprendizagem por subsidiar o desenvolvimento da competência informacional. Pense sobre as perspectivas da organização e divulgação de conhecimentos, meios de comunicação social-mente, multirreferencialidad ea necessidade de políticas públicas para promover uma melhor comunicação e informação alfabetização.

Palavras-chave: acesso ao conhecimento, alfabetização exigências do século XXI, a mediação, cognição

\footnotetext{
* Universidade Federal da Bahia (UFBA) / Instituto de Ciência da Informação (ICI), Brasil varela@ufba.br

** Universidade Federal da Bahia (UFBA) / Instituto de Ciência da Informação (ICI), Brasil marilene@ufba.br
} 
Title: Signification spaces and representation from cognitive theories, from mediation and from the multi-referential in the process of literacy is access to knowledge

\begin{abstract}
This article intends to analyze the man of the $21^{\text {th }}$ century differential situations in order to access information / knowledge, emphasizing the complexity to apprehend and to understand the reality in a context of continuous transformations in the scientific, technological, cultural, political, social and economic fields. It makes a review about illiteracy, literacy and cross culture concepts and the school and the school library function in the learning process subsidizing the development of the informational power. Reflects on new perspectives for the knowledge organization and dissemination, mediation, and multirange and the necessity of public policies in behalf of the informational and communicational literacy potentiation.
\end{abstract}

Keywords: knowledge access, literacy, $21^{\text {th }}$ century differential situations, disparity, mediation, cognition.

\title{
1 Introducción
}

El punto 20 de las metas del Milenio de la ONU - Organización de las Naciones Unidas - (2000) establece: "asegurar que todos puedan aprovechar de los beneficios de las nuevas tecnologías, especialmente la tecnología de la información y de la comunicación, en conformidad con las recomendaciones de la Declaración Ministerial del Consejo Económico y Social de 2000. "Actualmente, la exclusión del conocimiento, que retira la posibilidad de que las personas cambien sus vidas y participen democráticamente en las decisiones importantes para el pleno desarrollo del país. Las nuevas tecnologías de información y comunicación son requisitos fundamentales para la educación transformadora e inclusiva.

No se constituye factor predominante a la inclusión digital, solamente el acceso físico a las tecnologías de la información y la internet; son necesarios algunos requisitos, como un dominio de habilidades de aprehensión y comprensión de la información, teniendo en cuenta esta información como una estructura o una totalidad relativa que necesita una operación para averiguar la funcionalidad de sus dependencias internas o partes constituyentes. Por lo tanto, el proceso de aprehender y comprender los contenidos informativos involucran múltiples procesos cognitivos, mediación, contextualización, entre otros. 
Espacios de significación y representación de las teorías cognitivas, de la mediación y de la multirreferencialidad en el proceso de alfabetización hacía el aceso al conocimiento

El conocimiento es construido por la interacción continua llevada a cabo por los ciudadanos individualmente y validado por todos los ciudadanos colectivamente. Así, los conceptos, ideas, leyes, teorías, hechos, personas, historia, geografía, manifestaciones artísticas, medios de comunicación, ética, política, el gobierno, valores etc - traducidos en contenidos formales de las ciencias, las artes y la filosofía - se encuentran en un conjunto de condiciones esenciales para la construcción del conocimiento. (MEC / INEP / ENEM, 2007.)

El hombre del siglo XXI se enfrenta a situaciones que envuelven la necesidad de solucionar problemas. ¿Cómo saber o adquirir nuevos conocimientos? ¿Cómo interpretar e aprehender la realidad en un contexto de cambios continuos, en la ciencia, en la cultura, en lo político, en lo social y lo económico? ¿Cómo aprender a ser rescatándose y construyéndose como persona? ¿Cómo realizar acciones sobre una práctica que es impulsada por las tradiciones del pasado y el futuro que no es? ¿Cómo vivir en un contexto de tantas diversidades, diferencias y singularidades donde el respecto y el amor están presentes?

Reflexionar sobre nuevas perspectivas para la organización y difusión del conocimiento, la mediación, la multirreferencialidad y las políticas públicas, para la potenciación de la alfabetización informacional y la comunicacional, como también aborda la mediación como subsidiaria de la actividad científica, que es el papel preponderante de los profesionales que se ocupan de la recuperación, organización y difusión de información.

Se propone aquí, analizar las exigencias de nuevos tiempos para el acceso a los conocimientos, en términos de la cognición - competencias y habilidades - y la exclusión de una parte de la población brasileña a la luz de la investigación sobre la alfabetización y el analfabetismo.

\section{Acceso al conocimiento en el mundo contemporáneo}

Cada vez más se necesita que los sujetos sepan a aprender y a elegir lo que es conocer, comprender hechos y fenómenos, establecer relaciones interpersonales, analizar, reflexionar y actuar en esta nueva organización mundial. Comprender los fenómenos significa ser competente para formular hipótesis o ideas sobre las relaciones causales que los determinan. Para esto, es importante establecer relaciones e inferencias.

Delante de una situación-problema implica seleccionar, organizar, relacionar e interpretar datos para tomar decisiones. Para decidir es necesario un corte significativo de una realidad, a veces compleja, que puede verse de muchas maneras y que puede competir en facto- 
res que no siempre es posible dar prioridad a todos al mismo tiempo. (MEC / INEP / ENEM, 2007.)

En este contexto, se viven tiempos en que los países revisan sus modelos educativos, analizan y ponen en práctica reformas curriculares más adecuadas para satisfacer las demandas de la sociedad contemporánea; una sociedad que, en términos de conocimiento, está abierta a todas las posibilidades. Nuevos tiempos apuntan para el desarrollo de un conjunto de competencias y habilidades esenciales para que se pueda, de hecho, comprender y reflexionar sobre la realidad, participando y actuando en una sociedad comprometida con el futuro.

En esta concepción, se privilegia la noción de que existe un proceso dinámico de desarrollo cognitivo mediado por la interacción del sujeto con el mundo. En este sentido, la inteligencia es vista no como una facultad mental o como una expresión de las capacidades innatas, sino como una estructura de posibilidades crecientes para la construcción de estrategias básicas de acciones y operaciones mentales con las cuales se construyen los conocimientos. Por lo tanto, no sólo el maestro o la escuela puede integrarse al desarrollo y fortalecimiento de la lectura del mundo, así como los bibliotecarios y científicos de la información.

\section{Diferenciales en el acceso al conocimiento}

La pregunta que surge ahora es si el acceso a la escuela y la frecuencia son suficientes para garantizar la adquisición de competencias necesarias para la vida personal y profesional, bien como el desarrollo de ciudadanos conscientes de los derechos y deberes. Además, otra cuestión está relacionada con la forma de reparar la exclusión educacional de millones de ciudadanos que ya han superado la edad de la escolaridad y no han terminado la educación básica. Para reflexionar sobre ello, son presentados y analizados los resultados del Indicador de Alfabetização Funcional (INAF).

El INAF, creado e implementado por el Instituto Paulo Montenegro, difusor de las prácticas educativas innovadoras, y por la ONG Ação Educativa, que se ha hecho desde 2001 y aplicado por muestreo de 2000 personas, entre los 15 y los 64 años, residentes en las zonas urbanas y rurales de todas las regiones de Brasil. El INAF trabaja con la palabra "alfabetización" en información / letramento, que corresponden a la expresión en Inglés literacy, que se refiere a la condición de los individuos o grupos sociales que hacen uso de la lengua escrita. El término alfabetización considera los dos ámbitos: 1) alfabetización en información / letramento (procesamiento de informaciones verbales en diversos formatos; la escritura y comprensión) y 2) numeración (la capacidad de comprender y trabajar con conceptos matemáticos 
Espacios de significación y representación de las teorías cognitivas, de la mediación y de la multirreferencialidad en el proceso de alfabetización hacía el aceso al conocimiento

y las representaciones matemáticas usadas en situaciones de la vida cotidiana. Basado en el análisis de tareas, el INAF ha definido cuatro niveles de alfabetización:

- Analfabetismo: la condición de los que no consiguen realizar tareas que impliquen la simple lectura de palabras y frases, mismo que una parte de estos consiga leer los números que le son familiares (números de teléfonos, precios etc).

- Alfabetización en nivel rudimentario: la gente tiene capacidad para localizar información explícita en textos breves y familiares (como un anuncio o minúscula carta), leer y escribir números usuales y realizar operaciones sencillas como el manejo habitual de dinero para el pago de pequeñas cantidades o hacer medidas de longitud usando la cinta.

- Alfabetización en nivel básico: la gente puede considerarse analfabeta funcional, ya que leen y entienden textos de tamaño medio, ubican informaciones, incluso si es necesario llevar a cabo pequeñas inferencias, leen números en millones, resuelven problemas que impliquen en una secuencia de operaciones sencillas y también hay concepto de proporcionalidad. Muestran, sin embargo, limitaciones a la hora de las operaciones que requieren el mayor número de elementos, medidas o relaciones.

- Alfabetización en nivel pleno: la gente tiene habilidades para comprender e interpretar los elementos habituales de la sociedad alfabetizada: leen textos largos, relacionan sus partes, comparan e interpretan la información, distinguen hecho de la opinión, realizan inferencias y resúmenes. En cuanto a las matemáticas, resuelven problemas que requieren una mayor planificación y control, con la participación de porcentajes, proporciones y cálculo de área, además de interpretar las tablas de doble entrada, mapas y gráficos. (INSTITUTO PAULO MONTENEGRO, 2007.)

Los resultados del INAF / Brasil durante el período 2001-2007 muestran que hay que invertir en calidad, a fin de que la educación garantize aprendizajes que los ciudadanos necesitan para participar, con autonomía y responsabilidad en la sociedad contemporánea. El cuadro siguiente muestra la evolución del indicador para el Total Brasil en el período comprendido entre 2001 y 2007, recordando que, para el período 2001-2005, los promedios de nota se utilizan para garantizar la comparabilidad de los datos.

\begin{tabular}{|c|c|c|c|c|c|}
\hline \multicolumn{7}{|c|}{ INAF / BRASIL - Los cambios en el indicador } \\
\hline & $2001-2002$ & $2002-2003$ & $2003-2004$ & $2004-2005$ & 2007 \\
\hline Analfabeto & $12 \%$ & $13 \%$ & $12 \%$ & $11 \%$ & $7 \%$ \\
\hline Rudimentario & $21 \%$ & $26 \%$ & $26 \%$ & $26 \%$ & $25 \%$ \\
\hline
\end{tabular}




\begin{tabular}{|c|c|c|c|c|c|}
\hline Básico & $34 \%$ & $36 \%$ & $37 \%$ & $38 \%$ & $40 \%$ \\
\hline Pleno & $26 \%$ & $25 \%$ & $25 \%$ & $26 \%$ & $28 \%$ \\
\hline Puntuación media & 100 & 98 & 100 & 101 & 105 \\
\hline
\end{tabular}

Cuadro 1 - INAF / BRASIL - Los cambios en el indicador. Fuente: INAF / Brasil

Se observa que:

- la proporción de los brasileños de 15 a 64 años por el INAF clasificados como "analfabetos absolutos" estan disminuyendo en los últimos años, que asciende a 7\% en la última encuesta. Lo mismo sucede con la proporción de personas clasificadas como nivel de alfabetización rudimentaria, equivalente en 2007 a 25\% de la población en el grupo de edad considerados;

- Aún se puede observar un crecimiento continuo de nivel básico, que pasó de $33 \%$ en 2001 a $40 \%$ en 2007 ;

- el nivel pleno ha fluctuado alrededor de una cuarta parte de todos los brasileños, y en 2007 también indica una tendencia de mejoria.

Los resultados también muestran que ni siempre el nivel de educación garantiza el nivel de competencias que se espera. El análisis de los niveles de alfabetización por nivel de educación muestran la realidad de la situación de Brasil:

- $64 \%$ de los brasileños entre 15 y 64 años que estudiaron hasta el $4^{\circ}$ grado, alcanzan el nivel de alfabetización rudimentaria; tienen capacidad para ubicar informaciones explicitas en textos cortos o hacer sencillas operaciones de matemáticas;

- $12 \%$ del $64 \%$ pueden considerarse analfabetos absolutos en términos de habilidades de lectura / escritura, no descifran palabras y frases y tienen dificultades para hacer frente a los números en situaciones de la vida cotidiana, a pesar de haber estudiado cuatro años en una escuela primaria;

- aquellos que estudiaron del $5^{\mathrm{a}}$ a $8^{\circ}$ grado, sólo el $20 \%$ se pueden considerar plenamente alfabetizados, mientras que la mayoría se ha encuadrado al nivel básico de alfabetización. Teniéndose en cuenta que el $26 \%$ de los que completaron entre el $5^{\circ}$ a $8^{\circ}$ grado pertenecen al nivel rudimentario, con graves limitaciones en términos de sus habilidades de lectura / escritura y las matemáticas;

- mientras el $47 \%$ de los que estudiaron o están estudiando en la escuela secundaria, llegan al pleno nivel de alfabetización (100\% previsto para este grupo) y casi un $45 \%$ permanecen en el nivel básico; 
Espacios de significación y representación de las teorías cognitivas, de la mediación y de la multirreferencialidad en el proceso de alfabetización hacía el aceso al conocimiento

- solamente entre los que llegan o completan la educación superior es que prevalecen (74\%) los individuos con el pleno dominio de las competencias de lectura / escritura y matemáticas.

El INAF también retrata la distribución de los distintos niveles de alfabetización por el territorio brasileño permitiendo evidenciar contrastes y diferencias regionales: la población de la región Sur es la que tiene mayores niveles de alfabetización, con $71 \%$ de alfabetización funcional, y 1/3 de forma plena; en el extremo opuesto, la región Nordeste, es la más grande en contingente de analfabetos funcionales, lo que representa el $46 \%$ de la población entre 15 y 64 años; también hay una similitud en el perfil de la población de las regiones Sur y Surdeste (mayor concentración en nivel básico, un pequeño número de analfabetos y proporciones equivalentes entre los niveles rudimentario y pleno).

En confirmación a los indicadores del INAF, el Intituto Brasileiro de Geografia e Estatística (IBGE), muestra que el 11,4\% de las personas de más de 15 años son analfabetas y el $23,5 \%$ son analfabetos funcionales, o sea, son considerados alfabetizados, pero no tienen capacidad de leer e interpretar la información. De la población total de más de 10 años de edad, el $11,3 \%$ no tienen educación o tienen menos de un año de estudio y sólo el $26 \%$ tienen 11 (once) o más años de estudios (IBGE, 2004-2005). Las cifras del IBGE muestran que, en general, la población brasileña no tiene un alto nivel y probablemente no dominan las tecnologías de la información.

El INAF aún muestra que la mayoría de los alfabetizados en nivel rudimentario (29\%) y básico (16\%) no suelen leen libros o leen un sólo tipo de libro (42\%), en general, la Biblia o libros religiosos. Sólo entre las personas alfabetizadas en nivel pleno tienen una amplia mayoría de los lectores que diversifican sus intereses (33\% suelen leer dos géneros y el $34 \%$ tres o más géneros). En la búsqueda de enriquecer los datos, en las ediciones de 2003 y 2005 el INAF ha preguntado a los entrevistados si ya estuvieron en una biblioteca y dónde. Los resultados muestran que hubo una disminución en el número de personas que ya han estado en una biblioteca pública (de 59\% a 49\%), pero aumentó ligeramente en los que ya cuentan con biblioteca escolar (45\% a 49\%). Un análisis multivariado de datos del INAF demostró que la frecuencia de más de un tipo de biblioteca es un importante diferencial en los niveles de alfabetización.

Una nueva calidad debe ser construida, teniendo en cuenta las exigencias para el uso de la lectura, la escritura, las matemáticas y no sólo para la continuidad de los estudios, pero 
para participar de manera eficiente e independiente en el mundo del trabajo y en el ejercicio de la ciudadanía. Lo que se viene examinando arriba puede así ser resumido:

\begin{abstract}
"No es posible enfrentar la inclusión digital sin tener en cuenta la elevada tasa de analfabetismo. Aunque la lucha contra el analfabetismo en el país conte con la participación de varios sectores de la sociedad, los recursos del gobierno siguen siendo insuficientes, sin contar que los recursos humanos, a ejemplo de bibliotecarios aún son muy escasos, pues los profesionales en el área no trabajan directamente con la educación y con el bienestar social. La formación del bibliotecario es altamente calificada para cumplir con la función social que promueva la ciudadanía en las comunidades carentes. Pero pocos son los interesados en el área educacional y social, ya que no hay retorno sobre la remuneración y la infraestructura que se ofrece. "(BAPTISTA, 2006, p. 6)
\end{abstract}

\title{
4 Cognición, mediación, multirreferencialidad: reflexiones
}

\subsection{Cognición}

\begin{abstract}
"La cognición es un concepto amplio y extenso pues se refiere a las actividades mentales involucradas en la adquisición, procesamiento, organización y utilización del conocimiento. Los principales procesos que involucran el término cognición incluyen: detectar, interpretar, clasificar y recuperar la información; evaluar las ideas; inferir principios; deducir reglas; imaginar posibilidades, generar estrategias de fantasear y soñar (MUSSEN et al., 1988, p.210)
\end{abstract}

Basándose en los datos presentados por el Indicador de Alfabetización Funcional (INAF), es necesario examinar a la luz de algunos teóricos de la área de estudios de la enseñanza, centrándose en la cognición humana, para respaldar algunas explicaciones sobre el proceso de alfabetización / analfabetismo que viene ocurriendo en la educación brasileña.

Inicialmente, cabe señalar que la etapa de desarrollo cognitivo, corresponde a la Educación Básica (compuesta por la educación de los niños, Escuela Primaria y Escuela Secundaria), en Brasil, se caracteriza por el período de operaciones formales, caracterizado por la llegada del razonamiento hipotético-deductivo. Para Piaget (1987), al lograr ese período, los jóvenes tienen la oportunidad de considerar la realidad como un resultado de múltiples y exhaustivas posibilidades. El razonamiento puede ser ejercido en la actualidad sobre enunciados puramente verbales o sobre propuestas. La inteligencia se entiende, por lo tanto, como una adaptación, teniendo como función estructurar el universo de forma similar a la estructuración del medio ambiente por el organismo humano. La estructura mental y el conocimiento se construyen en una relación dialéctica entre la maduración biológica y el medio ambiente.

Basándose en estudios de Vygotsky (1991), la inteligencia se realiza a través de ciertas herramientas psicológicas que el sujeto encuentra en su entorno, incluido el lenguaje que es 
Espacios de significación y representación de las teorías cognitivas, de la mediación y de la multirreferencialidad en el proceso de alfabetización hacía el aceso al conocimiento

considerado como la clave. La actividad práctica que se involucra el sujeto sería incluido en las actividades mentales, cada vez más complejas, gracias a las palabras, fuente de la formación conceptual y del proceso de internalización.

Vygotsky (1991) da importancia al desarrollo del lenguaje, constituyéndose la palabra en rico instrumento para transmitir la experiencia histórica de la humanidad. Todo ser humano insertado en una realidad socio-histórica, sólo adquiere la condición humana si fuera en su relación con el mundo, mediada por medio de su cultura - signo, palabra, símbolo. El conocimiento es, por lo tanto, una producción cultural, directamente relacionado con el lenguaje y con la interacción social.

A lo largo del trabajo de Paulo Freire, son destacados elementos ontológicos / epistemológicos, entre ellos el concepto de la educación "dialógica" frente a la educación "bancaria”. En la educación bancaria se enfrentan dos estructuras: el educador que sabe y a del educando que no sabe y aprende, y en la educación dialógica, dos procesos de estructuración gnosiológica interactúan, un enseñando / aprendiendo con el otro (educador e educando) y en el otro proceso ocurre cuando educador y educando construyen el conocimiento del mundo. El estudiante reconoce que hay distintas realidades, descubre que existe yo y el yo de los otros, aunque hay diferentes órbitas existenciales; toma conciencia de su historicidad, es capaz de discernir y relacionarse con otros seres; llegar a ser sujeto por medio de las relaciones.

Según Morin (2000), un conocimiento pertinente debe hacer frente a la complejidad que se da cuando los distintos elementos constitutivos de un conjunto, son inseparables. Hay un tejido interdependiente, interactivo e inter-retroactivo del objeto de conocimiento con su contexto. El autor sugiere que la capacidad de aprender está vinculada al desarrollo de competencias innatas de las personas a adquirir conocimientos, vinculados a las influencias y estímulos externos, originados en la cultura.

Lo que se aprende, por lo tanto, es que los estudios sobre la cognición humana se caracterizan por ser un fenómeno multifactorial y altamente complejo, con la participación de diversas actividades mentales, en una interacción constante, lo que resulta en cambios de competencias del sujeto cognoscente.

\subsection{La mediación: mediar, mediándose}


Vygotsky (1991) hace hincapié en la relación de mediación cultural de las estructuras psicológicas. El factor decisivo en el desarrollo no recae sobre el individuo, sujeto de los procesos de construcción intelectual, sino en los procesos de mediación de las estructuras cognitivas y lingüísticas. La mediación es la acción que se interpone entre el sujeto y el objeto de aprendizaje, siendo la palabra de una importancia fundamental.

Discípulo de Piaget, Feuerstein desarrolló la Teoría de la Modificabilidad Cognitiva Estructural (TMCE), y propone conceptos en el ámbito de la cognición, enfatizando en el aprendizaje y el desarrollo humano. Feuerstein averigua como el sujeto llega a la solución del problema y propone el mapa cognitivo como una herramienta de análisis del acto mental. Explica el aprendizaje humano, no sólo por la integridad biológica de los genes y cromosomas, o simplemente por la exposición directa a los objetos, acontecimientos, actitudes y situaciones. El aprendizaje surge de una relación individuo - medio con la mediación de otras personas más experimentadas, cuyas prácticas y creencias culturales son transmitidas por la activación de zonas más amplias de desarrollo crítico y creativo, hacia a la autonomía cognitiva, debido a un aprendizaje mediado (Feuerstein, 1980).

Para explicar como la interacción humana impulsiona el desarrollo de la estructura cognitiva y fomenta la capacidad humana a la modificabilidad, Feuerstein desarrolló una estrategia metodológica denominada Experiencia de Aprendizaje Mediado (EAM), considerada como una interacción de los seres humanos y su contexto sociocultural. Se refiere a las experiencias que influyen en la "propensión a aprender" de la persona y a la calidad de la interacción que ayuda al estudiante a ser modificado mediante la exposición al estímulo hacia los altos y eficientes niveles de funcionamiento y adaptación (Feuerstein, 1991).

Cuando el profesor es el mediador, él aplica las estrategias para mediar en la presentación de las tareas, en la codificación / decodificación de los términos, en la construcción de los conceptos, en la preparación de un trabajo independiente, en la exploración de los procesos y estrategias, en la orientación temporal y espacial, en la producción de reflexión e interiorizado en la enseñanza de elementos específicos en la construcción de "puentes" con otras áreas de contenido y de la vida en general, con el objetivo de llegar a la generalización y a la abstracción conceptual. Sin embargo, el proceso de la mediación ya más allá de las fronteras de la escuela, está con la familia y otros profesionales que se ocupan de la información.

Para Paulo Freire (1979), la mediación es la acción a través de la cual el hombre llega a ser sujeto por una reflexión sobre su situación, acerca de su entorno. "Cuanto más el hombre reflite, más él surge y se compromete a intervenir en la realidad." Ya Morin (2000) observa que la mediación es el diálogo y la interacción de orden y el de-sorden en el mundo, tejiendo 
Espacios de significación y representación de las teorías cognitivas, de la mediación y de la multirreferencialidad en el proceso de alfabetización hacía el aceso al conocimiento

todas las partes, y el todo con las partes y entre las propias partes. "El conocimiento es, naturalmente, conectado a la vida, haciendo parte de la existencia humana. La acción de conocer se presenta en las acciones biológicas, cerebrales, espirituales, culturales, lingüísticas, sociopolíticas e históricas. "(Morin, 2000)

Se puede inferir que la mediación subsidia la actitud científica, cuando mediador y mediado trabajan las funciones cognitivas, desarrollando el curso de observación, de análisis y de trascendencia; entablan un diálogo, organizan el pensamiento para resolver situacionesproblema; analizan los éxitos y las dificultades para encontrar alternativas para resolver los problemas; vivencian la experiencia del análisis, llegando a la generalización, a la introspección, en un proceso de construcción del sistema metacognitivo; desarrollan la síntesis para llegar a conclusiones, generando principios y creando "puentes" y conexiones con el mundo exterior; vivencían la experiencia interdisciplinaria y la contextualización esencial para una actitud científica; ejercitan trayectorias cognitivas utilizando diferentes sistemas simbólicos; construyen abstracciones hasta llegar al más complejo.

\subsection{Múltirreferencialidad: la complejidad de los fenómenos sociales}

Considerando lo expuesto, para comprender el fenómeno de alfabetización / analfabetismo, se buscan contribuciones del enfoque multirreferencial que, a la medida que se pretende garantizar la complejidad de los fenómenos sociales, requiere, para su comprensión, la combinación de una serie de enfoques que integren las diferentes áreas del conocimiento.

Ardoino (1995) señala que la aparición del foco multirreferencial en el ámbito de las Ciencias Humanas está directamente relacionada con el reconocimiento de la complejidad y de la heterogeneidad que caracterizan a las prácticas sociales. La postura epistemológica de Ardoino se estructura a partir del reconocimiento del carácter pluralista de los fenómenos sociales, "significa, en lugar de buscar un sistema unitario explicativo, las ciencias humanas necesitan explicaciones, o de visiones, de perspectivas plurales para tener en cuenta un poco mejor, la complejidad de los objetos. "(Ardoino, 1998a).

Existe, pues, una perspectiva para la comprensión de los fenómenos sociales, considerándose la pluralidad / diversidad y la complejidad. Para Morin (1994), el paradigma de la complejidad no "produce" ni determina la inteligibilidad: "el puede incitar la estrategia / inte- 
ligencia del sujeto investigador a considerar la complejidad de la cuestión estudiada" (Morin, 1994). Esto es, para Morin, así como para Ardoino (1998b), la complejidad no esta en el objeto, sino en la visión que utiliza el investigador para estudiar su objeto, o sea, en la forma que el aborda los fenómenos.

Desde la perspectiva de la complejidad, la educación se entiende en el enfoque multirreferencial, "como una función global, que atraviesa el conjunto de los campos de las ciencias, del hombre y de la sociedad, interesando tanto al psicólogo social como al psicólogo, al economista, al sociólogo, al filósofo o al historiador y etc."(Ardoino, 1995, p. 7). A la medida que los fenómenos educativos son percibidos como fenómenos complejos, es necesario un enfoque que atienda a estas diferentes perspectivas, reconociendo sus recurrencias y contradicciones, de manera que ellas no se reduzcan entre si.

Así, reflexionar sobre el conocimiento y controlar los procesos cognitivos son los pasos que conducen a la formación de un receptor, que percibe las relaciones y la forma de relacionarse, descubriendo e infiriendo informaciones y significados por medio de estrategias cada vez más flexibles. Comprender información es un acto cognitivo y social, cuando dos sujetos (emisor y receptor) conocedores de una determinada realidad, interactúan.

\section{Mecanismos que desencadenan y dirigen la inteligencia en el proceso de lectura}

Cada acto de lectura es un acontecimiento, o una transacción que involucra a un determinado lector y un determinado patrón de signos, un texto que ocurre en un momento y contexto particular. En lugar de dos entidades fijas actuando una sobre otra, el lector y el texto son dos aspectos de una situación dinámica total. El "sentido" no esta en un texto "listo" o en un lector ya establecido, pero lo que pasa o llega a ser, tiene que ver con una operación que se da en el proceso entre el lector y el texto. "(Rosenblatt, 1998).

"Para comprender un texto", dijo Merlin C. Wittrock, "nosotros no sólo lo leemos en el sentido literal de la palabra: nosotros construimos un significado para él. Así procediendo, los lectores crean imágenes y transformaciones verbales para representar su significado y lo que es más impresionante: ellos generan sentidos a la medida que leen, construyendo relacio-

nes entre sus conocimientos, su memoria de experiencias, y las frases, párrafos y pasajes por escrito". (Wittrock apud Manguel, 1997, p. 54.)

Por su complejidad, el desciframiento de estas vías está aún lejos de haberse alcanzado, y por eso siguen siendo válidas las palabras del investigador americano E. B. Huey que a principios del siglo XX, ha admitido que la decifracción "sería el apogeo de la relación del 
Espacios de significación y representación de las teorías cognitivas, de la mediación y de la multirreferencialidad en el proceso de alfabetización hacía el aceso al conocimiento

psicólogo, pues seria describir muchos de los funcionamientos más complejos de la mente humana." (Apud HUEY Manguel, 1997, p. 56.)

Por medio de ejercicios constantes de lectura, de análisis y de comprensión de texto, se puede aumentar actitudes "descodificadoras", en relación con toda la realidad, o de una actitud creativa, fomentando en los lectores una especie de impulso exegético que se amplié al universo extra-textual y se convierte en el deseo de sentido. Así que, quizás, se pueda fomentar la capacidad de captar y comprender las interdependencias, la multiplicidad y la complejidad de lo real. Porque, definitivamente,

\begin{abstract}
"Siempre que el lector que lee el sentido, es el lector que da a un objeto lugar o evento una cierta claridad posible, que la reconoce en ellos, es el lector que debe otorgar significados a un sistema de signos y, a continuación, descifrarlos. Todos nosotros leemos a nosotros mismos y al mundo a nuestro alrededor para ver lo que somos y dónde estamos. Leemos para entender, o para comenzar a entender. No podemos dejar de leer. Leer, casi como respirar, es nuestra función esencial. "(Manguel, 1997, p.19-20.)
\end{abstract}

Basados en las investigaciones de la teoría literaria, de la lingüística, la antropología simbólica de la psicología cognitiva y de la psicología evolutiva, Bruner (1997) examina los actos mentales que se inserten en la creación imaginaria de mundos posibles, y muestra que la actividad del imaginario impregna, al mismo tiempo, las humanidades, la literatura y la filosofía, llegando incluso a la percepción de si mismo.

Para Bruner (1997), el pensamiento narrativo es aquel pensamiento que construye infinitos mundos posibles por medio del lenguaje, de las imágenes, de las invenciones y principalmente con la memoria autobiográfica, es la esencia de nuestra propia naturaleza. En Actos de Significación, el autor sostiene que la revolución cognitiva, como se había diseñado originalmente, incluía la posibilidad de que la psicología cooperase con la antropología, la lingüística, la filosofía y la historia, "incluso con los contenidos de derecho." (Bruner, 1997, p. 1617.)

Según Bakhtin (2002), la concepción del discurso corresponde a todo el enunciado que construye sentido, teniendo en cuenta las condiciones socio-históricas en la que se produce. Estas condiciones implican la comprensión de las formas de organización social que rigen y proponen las agendas de construcción del conocimiento y sus complicaciones en la organización del conocimiento. En ese caso, pensar en la organización del conocimiento y en los procesos de transferencia de la información demanda que se vean los procedimientos de producción del conocimiento. 
La construcción simbólica es socialmente determinada, debe comprender antes de cualquier otra cosa, las culturas en las que grupos sociales están insertados y como se identifican entre sí y se diferencian de los demás. En este juego de identidad y diferencia, se establecen procesos de representación, entre ellos, el metafórico, es decir, desde la propuesta de Lakoff y Johson (1980), el modo de la excelencia de la representación.

De todos modos, para que se pueda entender el fenómeno de la vida, que en sí mismo es la base que fundamenta la incesante, permanente y mutante búsqueda de la ciencia, es importante reconocer la total interdependencia entre el individuo con los demás y la naturaleza, porque mutuamente se complementan. En esa perspectiva, D'Ambrosio (2000) muestra que por medio de herramientas y de la tecnología, la especie humana establece diferentes vínculos entre el individuo y la naturaleza. Debido a la producción y trabajo del hombre, se crean intermediaciones entre la naturaleza y los demás y, a través de la comunicación y de sus emociones, las personas se integran y, a través de la intermediación, resulta el encuentro entre el comportamiento y el conocimiento y así se da la consciencia.

Cuando se trata explicar el comportamiento humano, se identifican dos grandes fuerzas que impulsan la vida: la supervivencia y la trascendencia. La primera es común a todas las especies y la segunda impulsa el desarrollo de un sentido a la propia existencia. Dan lugar a los conocimientos y definen el comportamiento, porque en la búsqueda de la supervivencia y de la trascendencia, se desarrolla la comunicación. (D'Ambrosio, 2000.)

\subsection{Alfabetismo / analfabetismo y la privación cultural}

El fenómeno de alfabetismo / analfabetismo, focado en la perspectiva del desarrollo cultural del sujeto, puede ser analizado a la luz de los estudios sobre la Privación Cultural, desarrollados por Reuven Feuerstein (1980). El concepto de Privación Cultural se basa en la ausencia de un tipo específico de transmisión cultural. Impide el desarrollo cognitivo y afectivo adecuado y reduce el grado de modificabilidad y de flexibilidad mental. La falta de un mediador o mediadores que se plantean entre el sujeto y el mundo, seleccionando y organizando las informaciones contextualizándolas culturalmente, provoca la denominada síndrome cultural.

El bajo funcionamiento cognitivo, el bajo rendimiento informacional o la descalificación de algunos sectores de la población, como grupos étnicos o grupos potencialmente excluidos, no son, según Feuerstein (1980), sinónimo de una cultura inferior. Es posible que la 
Espacios de significación y representación de las teorías cognitivas, de la mediación y de la multirreferencialidad en el proceso de alfabetización hacía el aceso al conocimiento

cultura de un pueblo sea muy rica económicamente, y su capacidad de información, sin embargo, sea muy baja, con altos porcentajes de fracaso y múltiples dificultades de aprendizaje, provocando problemas de inadecuación social.

Feuerstein (1980) señala que muchas personas están "alienadas" en relación a su propia cultura, como resultado de factores sociales, psicofísicos, educacionales y ambientales, que es una ruptura en la transmisión cultural. No se limite a hablar de las diferencias culturales definidas como comportamiento deficiente, debido a la falta de familiaridad con las tareas de aprendizaje y estilos cognitivos, o con los contenidos y modalidades de presentación.

El antagonismo entre la necesidad de la dependencia para sobrevivir y la necesidad de independencia para la realización personal y existencial caracterizan el conflicto educacional que ocurre en la actualidad en nuestra sociedad. Un individuo socializado en una cultura minoritaria es culturalmente diferente de los miembros de la cultura dominante y pueden diferir de los otros en rasgos culturales.

Así, las múltiples facetas del fenómeno de la alfabetización, la variedad y heterogeneidad de dimensiones, la diversidad de las relaciones con la sociedad y la cultura llevan a la conclusión de que no sólo es imposible formular un concepto general y universal del fenómeno, pero también son las innumerables perspectivas teóricas y metodológicas, según las cuales uno puede ver eso. Las perspectivas ora privilegian la dimensión social, ora la dimensión individual, ora una faceta, ora otra, a ejemplo de la perspectiva histórica, antropológica, sociológica, psicológica, sociolingüística, lingüística, discursiva, textual, literaria, educativa y política, es decir, la multirreferencialidad entendida como una pluralidad de opiniones al respecto de una realidad y una pluralidad de lenguajes a fin de reflejar esta realidad y las visiones direccionadas a ella.

\section{Nuevas perspectivas para la organización y difusión del conocimiento}

Cabe señalar que los primeros estudios sobre el uso de la información se registran al final de los años 40 del siglo pasado, aunque los estudios se dedicaban al comportamiento de los científicos y tecnólogos en el uso de la literatura científica y los canales de información.

En los últimos 25 años con la expansión de las tecnologías de la información y la comunicación, los estudios se han centrado con más intensidad a la comprensión de los tipos de comportamiento informacional humano. El campo de la conducta informacional humana se refiere a conceptos tales como: los contextos de información, necesidades de información, 
comportamientos de búsqueda de información, los modelos de acceso a la información, recuperación, difusión, transformación y uso de la información. Todo esto en la creencia de que la información es esencial para el funcionamiento y la interacción de los individuos, grupos sociales, organizaciones y empresas, así como el potencial de transformar el conocimiento y apoyar las decisiones y acciones (TODD, 2003).

Es una creciente la literatura sobre el comportamiento informacional en diversas partes del mundo. Tres líneas de investigación se presentan: la biblioteca escolarsubvencionando el aprendizaje, el comportamiento de la gente y la Internet, el comportamiento de búsqueda de información para los temas cotidianos. Estas experiencias suceden interrelacionadas y / o simultáneamente.

Encuestas muestran que la biblioteca escolar se ha presentado como un agente clave en el proceso de aprendizaje, si su propuesta está integrada en el currículo escolar. Se presenta como un elemento importante en el desarrollo de competencias informacionales que implica a varias habilidades, contribuyendo directamente a la formación crítica y reflexiva de los sujetos. Aunque las encuestas sean avanzadas, aún no están claros los efectos reales de los programas de la biblioteca escolar en el aprendizaje de los estudiantes y la forma en que pueden ser medidos y evaluados en la práctica. Como ejemplo, fueran seleccionados las siguientes experiencias:

a) El estudio de Baughman (2000) señala que en el estado de Massachusetts, el $92 \%$ de las escuelas públicas tienen bibliotecas escolares. Los ítems evaluados fueron: datos personales, colecciones de libros, infraestructura tecnológica y retirada de los libros en las bibliotecas. Se demostró que las puntuaciones de los estudiantes son más altas donde existen escuelas con programas para las bibliotecas y que presentan un buen número de libros por estudiante con un intenso uso, con flexibilidad de horarios y programas de instrucción. Bibliotecas donde hay una mayor inversión de materiales por alumno, donde los estudiantes son atendidos por un bibliotecario en tiempo completo con la ayuda de personal especializado, donde hay colecciones automatizadas y cuando además la biblioteca se ajusta con la estructura curricular de la escuela, subvencionan, sin duda, el fortalecimiento del proceso de enseñanzaaprendizaje.

b) En Australia, Lonsdale (2003) preparó un importante informe sobre el tema, publicado por el Australian Council for Educational Research. El documento sugiere que las bibliotecas escolares, para tener efectos positivos en actividades estudiantiles, deberán tener las siguientes características: un programa potente de biblioteca con una buena asistencia, planificado y fundamentado para llevar a la mejora de la actividad para el estudiante, independien- 
Espacios de significación y representación de las teorías cognitivas, de la mediación y de la multirreferencialidad en el proceso de alfabetización hacía el aceso al conocimiento

temente de los niveles educativos o socioeconómicos de adultos en la comunidad; una potente red informática que conecta los recursos de la biblioteca a las aulas y laboratorios; la calidad de la colección; fomentar una mayor utilización de la biblioteca de la escuela; relaciones de colaboración entre los profesores y los bibliotecarios escolares, especialmente en relación con la planificación de las unidades de instrucción, desarrollo de colecciones, y la garantía para la asignación del desarrollo de los profesores. Se registra que: a) cuanto más rica colección de impresos, más incentivos existen para la lectura, lo que culmina con el desarrollo de la comprensión, el crecimiento en el vocabulario, habilidades de ortografía, la gramática y el estilo de escritura, b) la integración de las habilidades informacionales al currículo escolar puede ofrecer a los estudiantes dominio sobre el terreno de los contenidos y de las habilidades en la búsqueda de información, c) las bibliotecas pueden hacer una diferencia positiva en la autoestima de los alumnos, en el sentimiento de confianza, independencia y sentido de la responsabilidad en su propio aprendizaje.

Estudios sobre la búsqueda de información en Internet apuntan a la falta de preparación, de habilidades específicas de los niños, niñas y adolescentes para hacer frente a las incompatibilidades e inconsistencias de la red mundial. La sobrecarga informacional de la red es una realidad que a menudo produce sentimientos de frustración, irritación, ansiedad, confusión y estrés. Hay variaciones significativas en el grado de éxito personal para obtener informaciones en Internet, esto se debe principalmente a la complejidad de las tareas en el nivel de conocimiento de cada persona y al método utilizado para llevar a cabo la búsqueda. Es necesario desarrollar habilidades específicas que permitan a la población obtener de manera exitosa la información disponible en Internet, que puede producirse a través de cursos y formación específica. Para accesar la información, las personas deben estar dispuesta a trabajar de manera adecuada y ser capaz de discernir en que informaciones confiar, o lo que es relevante o no.

Cuanto a la investigación sobre la búsqueda de informaciones para temas cotidianos y personales que subsidien decisiones personales sobre diversos intereses (drogas, sexo, profesión, etc.), los estudios sugieren que algunas veces los estudiantes se sienten ansiosos y no muy bien servidos en sus necesidades. En este caso, las sugerencias son para que las bibliotecas y servicios de información deban anticiparse a sus necesidades y buscar disponibilizar las informaciones de manera más accesible y atractivo, ya que, a menudo, los usuarios no saben como expresarse adecuadamente a sus necesidades, o se sienten avergonzados, pero tienen que tomar decisiones y quieren construir sus propios conceptos y opiniones. 
Según Todd (2003), un aspecto consistente que se desprende de todos estos estudios es la necesidad de desarrollar competencias informacionales y críticas en los estudiantes, desarrollando las bases intelectuales para que puedan utilizar correctamente la información, cuestionarla, criticarla y accederla de forma exitosa en sus necesidades de aprendizaje, y lograr los resultados deseados del conocimiento y la capacidad para conducir y utilizar con eficacia la cantidad de información con que se enfrentan en sus preguntas para la construcción de nuevos conocimientos.

En un nivel más específico, Todd (2003) afirma que estas competencias pueden permitir mejoras en los sitios de la Web y de instigar las cuestiones importantes, tales como: ¿Qué puede hacer este sitio para mí? ¿De quien son los intereses atendidos que este texto está tratando de decir? ¿Cuáles son las posibilidades de significado de este texto? ¿Lo que se acerca de esta información y cómo se relaciona con este sitio? ¿Cómo se relaciona este conocimiento a otros sitios Web y otras fuentes de información? ¿Cuáles son las alternativas y puntos de vista opuestos y donde podría encontrarlos? ¿Cómo que este sitio puede ayudarme a construir una posición alternativa? ¿Con quién puedo hablar acerca de él? ¿Qué voces son silenciadas aquí? ¿Qué medidas puedo tomar?

\section{Conclusión}

Diferentes paradigmas coexisten en la comunidad científica contemporánea. En una realidad cada vez más compleja que identifica el mundo como una sociedad del conocimiento, las antiguas certezas, la causalidad, la objetividad del conocimiento neutoniano, cartesiano o positivista permitieron nuevas formas de conocer. Las revolucionarias condiciones de búsqueda y difusión de la información por medio de infoways, especialmente por la Internet, han llevado a la aparición de nuevas formas de conocimiento. La rapidez, la velocidad, la aceleración de la investigación, crearan una nueva orden en la ciencia en la que se permite el aumento de la participación del sujeto en la comprensión del fenómeno que está investigando.

La integración de la Ciencia de la Información con las Ciencias Cognitivas ocurre con el fin de comprender los procesos cognitivos envolvidos en el comportamiento de los usuarios de los sistemas, de productos y de servicios de información. Esto se observa cuando la Ciencia de la Información intenta describir las formas por medio de las cuales los individuos recopilan, seleccionan y utilizan la información. La idea que esta detrás de los estudios sobre el uso de la información y del usuario es que la información es el elemento generador de la solu- 
Espacios de significación y representación de las teorías cognitivas, de la mediación y de la multirreferencialidad en el proceso de alfabetización hacía el aceso al conocimiento

ción de los problemas relacionados con el medio ambiente en el cual los usuarios actúan. La resolución de estos problemas conduce al usuario a modificar, o más bien, para aumentar su caudal de conocimientos, porque la información es el elemento que genera el conocimiento en el individuo.

La idea básica que subsidia los estudios de la Ciencia de la Información es la que el conocimiento tiene lugar cuando la información es percibida y aceptada, y siendo toda la alteración provocada en el caudal mental del saber del sujeto.

Los debates propuestos en este artículo muestran que la búsqueda y la utilización de la información son necesarios para la producción de conocimiento, que a su vez demanda competencias cuyo desarrollo exige una formación especial, denominada Alfabetización Informacional. Por lo tanto, parece que ya no permite hacer caso omiso de la Biblioteca. La Ciencia de la Información también tiene que hacer frente de manera más directa en el aprendizaje, porque la intención de gestionar la información es para hacerla accesible a las personas. Hay que considerar los aspectos semánticos (cognitivo) y pragmático (real), incluyendo las propiedades, por lo tanto, tanto en el contenido y en el significado acerca de la función social, al ver la posibilidad de transformar las estructuras del conocimiento, el conocimiento visto como algo provisorio y permanente revisión. Por coincidencia, varios fenómenos dentro de la escuela interfieren en el proceso informacional de la colección a la recuperación de información, comprometiendo su uso en la producción de conocimientos. La mayoría de ellos no es, sin embargo, el resultado de las políticas locales en materia de educación, sino una "crisis general que afectó al mundo moderno en todas partes y en casi todas las esferas de la vida." (Arendt, 2000, p. 221.)

Se evidencia que la inclusión social en el mundo contemporáneo, cuyas bases de producción se basan en el conocimiento y las tecnologías de la información, se hace necesario que algunas condiciones como la plena alfabetización, la competencia informacional e información multirreferencial. Los estudios económicos sobre el mundo del trabajo y el empleatismo han mostrado que hay mucho trabajo, pero hay falta de personal con experiencia para atender la demanda de la sociedad.

En este sentido, el Brasil tiene que fortalecer y desencadenar políticas públicas que se ocupan de la capacitación plena de personas y también fomentar el acceso a la información, creando las condiciones físicas de acceso a la información cultural, a la científica y tecnológica. 


\section{Referencias}

ARDOINO, J. Multiréferentielle (analyse). On: ARDOINO, J. Lê directeur et l'intelligence de l'organization : Repéres et notes de lecture. Ivry: ANDES, 1995. p.7-9.

ARDOINO, J. Abordagem multirreferencial (plural) das situações educativas e formativas. In: BARBOSA, M. G. (coord.). Multirreferencialidade nas ciências e na educação. São Carlos : Editora da UFSCar, 1998a, p.24-41.

ARDOINO, J. Abordagem Multirreferencial: a epistemologia das ciências antropo-sociais. 1998b. Palestra proferida na Pontifícia Universidade Católica do Rio Grande do Sul - Faculdade de Psicologia, no dia 14/10/1998.

ARENDT, Hannah. A Condição Humana. 10a . ed. Rio de Janeiro: Ed. Forense Universitária, 2000 .

BAKHTIN, M. (VOLOCHÍNOV). Marxismo e Filosofia da Linguagem. (1 a edição 1929). Tradução: Michel Lahud e Yara Frateschi Vieira. São Paulo: Editora HUCITECANNABLUME, 10 $0^{\mathrm{a}}$ edição, 2002.

BAPTISTA, S. G. A inclusão digital: programas governamentais e o profissional da informação - reflexões. Revista Inclusão Social, Brasília, v. 2, n. 2, 2006.

BAUGHMAN, James C. School libraries and MCAS scores. 2000. In: FIALHO, Janaína Ferreira. ANDRADE, Maria Eugênia Albino. Comportamento Informacional de Crianças e Adolescentes : uma revisão da literatura estrangeira". Ciência da Informação. v. 36, n.1. p. 20-34, jan-abr/2007. Acessado em: 24/11/2008.

BELKIN, Nicholas J. "The cognitive viewpoint in information science". 1990. In: FIALHO, Janaína Ferreira. ANDRADE, Maria Eugênia Albino. "Comportamento Informacional de Crianças e Adolescentes : uma revisão da literatura estrangeira”. Ciência da Informação. v. 36, n. 01. pág. 20-34, jan-abr/2007. Acessado em: 24/11/2008.

BRUNER, Jerome. Atos de significação. Trad. Sandra Costa. Porto Alegre: Artes Médicas, 1997.

D’AMBROSIO, Ubiratan. Uma visão transdisciplinar. Pátis : Revista Pedagógica. Porto Alegre, ano 4, n. ${ }^{\circ} 13$, p. 17-20, mai-jul, 2000.

FEUERSTEIN, Reuven. Instrumental Enrichment: an Intervention Program for Cognitive Modifiability. Baltimore: University Park Press, 1980. 
Espacios de significación y representación de las teorías cognitivas, de la mediación y de la multirreferencialidad en el proceso de alfabetización hacía el aceso al conocimiento

FEUERSTEIN, Reuven. "Libertad y realización personal”. El Mercurio, Artes y Letras, Santiago do Chile, 1991.

FREIRE, Paulo. Educação e mudança. Rio de Janeiro : Paz \& Terra, 1979.

HUEY, E. B. The psycology and pedagogy of reading. 1908. In: MANGUEL, Alberto. Uma história da leitura. Trad. Pedro Maia Soares. São Paulo: Cia das Letras, 1997.

INSTITUTO PAULO MONTENGRO - IPM. Indicador de Alfabetismo Funcional (INAF): Relatório 2007. São Paulo: IPM, 2007.

LAKOFF, George; JOHNSON, Mark. Metraphors we live by. Chicago: The University of Chicago Press. 242p, 1980.

LONSDALE, Michele. "Impact of school libraries on student achievement: a review of the research". 2003. In: FIALHO, Janaína Ferreira. ANDRADE, Maria Eugênia Albino. "Comportamento Informacional de Crianças e Adolescentes : uma revisão da literatura estrangeira. Ciência da Informação". v. 36, n. 01. pág. 20-34, jan-abr/2007. Acessado em: 24/11/2008.

MANGUEL, Alberto. Uma história da leitura. Trad. Pedro Maia Soares. São Paulo: Cia das Letras, 1997.

MEC/Inep. ENEM (Exame Nacional do Ensino Médio): Relatório pedagógico 2007. Brasília: MEC/Inep, 2007.

MORIN, Edgar. Ciência com consciência, Lisboa: Publicações Europa-América, 1994.

MORIN, Edgard. Os sete saberes necessários à educação do futuro. Trad. Catarina Eleonora F. da Silva e Jeanne Sawaya; ver. Téc. Edgard Assis Carvalho. São Paulo / Brasília : Cortez / Unesco, 2000.

MUSSEN, P. H.; CONGER, J. H.; KAGAN, J.; HUSTON, A. C. Desenvolvimento e personalidade da criança. São Paulo : Harbra, 1988.

OLMI, Alba; PERKOSKI, Norberto (orgs.). Leitura e cognição: uma abordagem transdisciplinar. Santa Cruz do Sul : EDUNISC, 256p, 2005. 
PIAGET, Jean. O nascimento da inteligência na criança. Rio de Janeiro : Guanabara, 1987. ROSENBLATT, Louise M. "The transactional theory of reading and writing". 1998. In: RUDELL B.; ROBERT et al. (Ed.). Theoritical models and processes of reading. Newark: International Reading Association.

SOARES, Magda. Alfabetização e letramento. $5^{\text {a }}$ Ed. São Paulo : Contexto, 2007.

TODD, Ross J. "Adolescents of the information age: patterns of information seeking and use, and implications for information professionals". 2003. In: FIALHO, Janaína Ferreira.

ANDRADE, Maria Eugênia Albino. "Comportamento Informacional de Crianças e Adolescentes : uma revisão da literatura estrangeira. Ciência da Informação”. v. 36, n. 01. pág. 2034, jan-abr/2007. Acessado em: 24/11/2008.

VIGÓTSKI, L. S. A formação social da mente. São Paulo: Martins Fontes, 1991.

WITTROCK, Merlin C. "Reading comprehension". 1981. In: MANGUEL, Alberto. Uma história da leitura. Trad. Pedro Maia Soares. São Paulo: Cia das Letras, 1997. 